\title{
L’organisation générale de la radioprotection au Sénégal
}

\author{
P. Casanova ${ }^{1 \star}$, M. Ndiaye ${ }^{2}$, A.S. Ndao ${ }^{3}$ et M.L. Sow ${ }^{2}$ \\ ${ }^{1}$ CNRS, UMI 3189 Environnement, santé, sociétés, Faculté de médecine secteur nord, 51 boulevard Pierre Dramard, 13916 Marseille \\ Cedex 20, France. \\ 2 UCAD, Faculté de médecine, Service de médecine légale et du travail, Dakar, Sénégal. \\ ${ }^{3}$ UCAD, Institut de technologie nucléaire appliquée, Dakar, Sénégal.
}

Reçu le 22 juin 2015 - Accepté le 2 août 2015

Résumé - L'organisation de la radioprotection au Sénégal est régie par trois textes fondamentaux qui en définissent les principes généraux et mettent en place les moyens juridiques de leurs actions. Un contrôle efficient des activités nucléaires pour assurer la protection des travailleurs, de l'environnement et des patients contre les rayonnements ionisants est assorti de sanctions pénales en cas d'infraction à cette législation.

\begin{abstract}
General organization of radiation protection in Senegal. Organization of radiation protection in Senegal is governed by three main texts that define the general principles and implement legal means for their actions. Efficient control of nuclear activities to ensure protection of workers, the environment and patients against ionizing radiation is subject to criminal penalties in case of breach of this legislation.
\end{abstract}

Keywords: radiation protection / safety standard / risk assessment / exposure / occupational / harmonization

\section{Introduction}

La loi n² 2001-01 du 3 janvier 2001 relative à la protection contre les rayonnements ionisants, aujourd'hui abrogée, a été le texte précurseur de l'organisation de la radioprotection au Sénégal (Gouvernement du Sénégal, 2001).

Auparavant, un arrêté du 20 avril 1956 du Gouverneur général de l'Afrique occidentale française concernant la protection du personnel exposé aux rayons $\mathrm{X}$ et au rayonnement du radium avait ouvert la voie (Gouverneur général de l'Afrique occidentale française, 1956).

La convention de Vienne de 1986 sur la notification rapide d'un accident nucléaire a été ratifiée en 2008 (Gouvernement du Sénégal, 2008).

Trois textes fondent aujourd'hui les bases légales de la radioprotection. La loi $\mathrm{n}^{\circ}$ 2004-17 du 15 juin 2004 relative à la protection contre les rayonnements ionisants pose les règles fondamentales (Gouvernement du Sénégal, 2004). La loi $n^{\circ}$ 2009-14 du 2 mars 2009 relative à la sécurité en matière nucléaire et à la radioprotection la complète en définissant en particulier l'autorité de sûreté nucléaire et l'élargit aux installations nucléaires de base (Gouvernement du Sénégal, 2009). Enfin, le décret n 2010-893 du 30 juin 2010 portant sur l'organisation de l'Autorité de Radioprotection et de

^ philippe.casanova@thalesgroup.com
Sûreté Nucléaire termine l'édifice juridique (Gouvernement du Sénégal, 2010).

Un projet de décret qui devrait être promulgué prochainement, mettra en œuvre les recommandations de la Commission Internationale de Protection Radiologique sur la protection des travailleurs. Il fera l'objet d'une étude séparée lors de sa publication.

\section{Les grands principes}

La loi de 2001 définit précisément le vocabulaire dans le domaine de la radioprotection qui comprend les accidents, les doses, les sources, les déchets, les installations et les unités. Ce préalable permettant de bien clarifier le texte demeure indispensable à une bonne application des principes, quand on sait à quel point l'émotion est présente et fait concurrence à la raison dès qu'on évoque ce sujet, dont la complexité technique rajoute encore à la difficulté du débat.

La loi de 2009 change de dimension en définissant les installations nucléaires de base. Il s'agit là d'une question plus politique que technique dans la mesure où le gouvernement de l'époque envisageait, pour combler les besoins énergétiques du pays, la construction d'une centrale nucléaire de production d'électricité. Ce projet est pour l'instant reporté sine die et la plupart des dispositions de la loi sans objet. 
La loi régit les activités liées à l'utilisation des matières nucléaires et des sources de rayonnements ionisants dans tous les domaines économiques et sociaux, publics et privés. Dans un but de protection, elle conditionne ces activités à des procédures claires. Les sources artificielles et naturelles sont concernées dans toutes les étapes allant de la production au traitement. De même sont concernées les sources provenant d'appareils électriques fonctionnant avec une différence de potentiel supérieure à $5 \mathrm{kV}$.

Cependant, elle ne s'applique pas à l'exposition au radon dans les habitations, aux radionucléides dans le corps humain ni au rayonnement cosmique existant au niveau du sol.

\section{Les moyens}

Un régime d'interdictions et d'obligations est mis en place. L'addition de substances radioactives dans les denrées alimentaires, les produits cosmétiques et les produits à usage domestique ou privé et les jouets font l'objet d'une interdiction. Il en est de même de l'importation et l'exportation de telles substances, d'appareils de radioscopie à des fins diagnostiques et de l'importation de déchets. Enfin, le principe de justification devient une obligation préalable.

Un système d'autorisation et de déclaration permet de déroger à l'interdiction. La déclaration doit être faite préalablement par toute personne physique ou morale détentrice d'une source à l'Autorité de sûreté nucléaire, à partir d'un certain seuil, par exemple un débit de dose de $1 \mathrm{mSv} \mathrm{h}^{-1}$ à $0,1 \mathrm{~m}$ de sa surface accessible pour un tube cathodique fonctionnant avec une différence de potentiel inférieure à $30 \mathrm{kV}$.

L'autorisation est obligatoire pour les sources d'irradiations industrielles, la radiothérapie, le radiodiagnostic, la médecine nucléaire, la production et le stockage de radionucléides, et les centres de recherche. Un dossier d'évaluation de l'impact, contenant les mesures à prendre pour la protection des travailleurs, du public et des patients, ainsi que la liste nominative et les qualifications en radioprotection des personnes utilisant les sources sont requis.

Une obligation de planification et de mise en œuvre des interventions est également prévue. Un plan d'urgence définit les responsabilités sur le site et à l'extérieur du site. Il prévoit les scénarios possibles, les acteurs en présence, les mesures de prévention, les méthodes d'évaluation des expositions et leurs conséquences ainsi que des exercices réguliers. Il doit être tenu à jour de façon périodique. La mise en œuvre implique l'information immédiate de l'Autorité de sûreté nucléaire sur la situation réelle et son évolution prévue, les mesures prises et les expositions subies et attendues.

\section{Le contrôle}

L'Autorité de Radioprotection et de Sûreté Nucléaire (ARSN) est un établissement public à statut spécial doté de la personnalité juridique et de l'autonomie financière. Elle est placée sous l'autorité du Premier ministre. Elle a un double rôle de contrôle de l'application de la réglementation et d'information des citoyens. Elle apporte son concours sur toutes les questions de sûreté nucléaire. Elle assure une mission de service public, peut donner des injonctions et édicter des règlements et des guides.

Elle élabore et propose au chef de l'État la politique nationale et la réglementation. Elle délivre les autorisations individuelles, inspecte les sites et les installations, fixe et perçoit des taxes, assiste le gouvernement, prend des mesures de coercition (mises en demeure) et coopère avec les organisations internationales.

Elle établit un rapport annuel qu'elle transmet au Président de la république, au Premier ministre et à l'Assemblée nationale.

Elle a, à sa tête, un Directeur général, nommé par décret et qui est assisté par un comité d'experts. Le Directeur général désigne, après avis du comité, les inspecteurs de sûreté nucléaire, les inspecteurs de radioprotection et les agents chargés du contrôle.

Le Comité des experts comprend cinq membres nommés pour une durée de cinq ans. Ces experts doivent être des spécialistes dans les domaines des sciences et techniques nucléaires, du droit, de l'environnement, de la radioprotection et de l'énergie. Un Président du comité est nommé par décret parmi les membres.

Le personnel est composé de fonctionnaires, d'agents contractuels et d'agents détachés par des établissements publics.

\section{Les sanctions}

Des peines d'emprisonnement de 1 à 5 ans et des amendes de 1 à 10 millions de francs CFA (1500 à $15000 €)$ sont prévues en cas d'infraction. De même, les personnes morales, à l'exception toutefois des personnes publiques, peuvent être poursuivies et condamnées. La cessation du danger peut être ordonnée sous astreinte, ainsi que la fermeture du site.

\section{Conclusion}

Le Sénégal s'est doté de textes simples et adaptés à la situation locale, exception faite d'une partie de la loi de 2009 sur les INB. L'ARSN bénéficie d'une réelle autonomie, mais ne constitue pas une autorité administrative indépendante. Elle dispose d'un site internet qui est un moyen efficace d'information du public. Les moyens de contrôle reposent sur un corps d'inspection.

La création de commissions d'information composées d'associations de citoyens serait de nature à améliorer la transparence et l'acceptabilité du nucléaire par la population.

\section{Références}

Gouvernement du Sénégal (2001) Loi n 2001-01 du 3 janvier 2001 relative à la protection contre les rayonnements ionisants, Journal officiel du Sénégal.

Gouvernement du Sénégal (2004) Loi n 2004-17 du 15 juin 2004 abrogeant les dispositions de la loi n ${ }^{\circ} 2001-01$ du 3 janvier 2001, Journal officiel du Sénégal.

Gouvernement du Sénégal (2008) Loi n 2008-52 du 24 septembre 2008 autorisant le Président de la république à ratifier la 
Convention sur l'assistance en cas d'accident nucléaire ou de situation d'urgence radiologique, adoptée par la Conférence générale de l'Agence Internationale de l'Énergie Atomique à Vienne le 26 septembre 1986, Journal officiel du Sénégal.

Gouvernement du Sénégal (2009) Loi n 2009-14 du 2 mars 2009 relative à la sécurité en matière nucléaire et à la radioprotection, Journal officiel du Sénégal.

Gouvernement du Sénégal (2010) Décret $\mathrm{n}^{\circ}$ 2010-893 du 30 juin 2010 portant organisation et fonctionnement de
l'Autorité de radioprotection et de sûreté nucléaire, Journal officiel du Sénégal.

Gouverneur général de l'Afrique occidentale française (1956) Arrêté 8409 IGTLS-AOF du 20 avril 1956 concernant la protection du personnel exposé aux rayons $\mathrm{X}$ et au rayonnement du radium dans les hôpitaux, les cliniques, les dispensaires, les cabinets médicaux, les cabinets dentaires et radiologiques, dans les maisons de santé et les centres anticancéreux, Journal officiel de l'Afrique occidentale française, 5 mai 1956, pp. 779-784.

Cite this article as: P. Casanova, M. Ndiaye, A.S. Ndao, M.L. Sow. L'organisation générale de la radioprotection au Sénégal. Radioprotection 50(4), 255-257 (2015). 